\title{
$\alpha 4^{\star}$ Nicotinic Receptors in preBötzinger Complex Mediate Cholinergic/Nicotinic Modulation of Respiratory Rhythm
}

\author{
Xuesi M. Shao, ${ }^{1}$ Wenbin Tan, ${ }^{1}$ Joanne Xiu, ${ }^{2}$ Nyssa Puskar, ${ }^{2}$ Carlos Fonck, ${ }^{2}$ Henry A. Lester, ${ }^{2}$ and Jack L. Feldman ${ }^{1}$ \\ ${ }^{1}$ Department of Neurobiology, David Geffen School of Medicine at University of California, Los Angeles, Los Angeles, California 90095-1763, and ${ }^{2}$ Division \\ of Biology, California Institute of Technology, Pasadena, California 91125
}

\begin{abstract}
Acetylcholine and nicotine can modulate respiratory patterns by acting on nicotinic acetylcholine receptors (nAChRs) in the preBötzinger complex (preBötC). To further explore the molecular composition of these nAChRs, we studied a knock-in mouse strain with a leucine-to-alanine mutation in the $\mathrm{M} 2$ pore-lining region ( $\left.\mathrm{L}^{\prime} \mathrm{A}\right)$ of the $\mathrm{nAChR} \alpha 4$ subunit; this mutation renders $\alpha 4$-containing receptors hypersensitive to agonists. We recorded respiratory-related rhythmic motor activity from hypoglossal nerve (XIIn) and patch-clamped preBötC inspiratory neurons in an in vitro medullary slice preparation from neonatal mice. Nicotine affected respiratory rhythm at concentrations $\sim 100$-fold lower in the homozygous L9' A knock-in mice compared with wild-type mice. Bath application of $5 \mathrm{~nm}$ nicotine increased the excitability of preBötC inspiratory neurons, increased respiratory frequency, and induced tonic/seizure-like activities in XIIn in L9' A mice, effects similar to those induced by $1 \mu \mathrm{m}$ nicotine in wild-type mice. In L9'A mice, microinjection of low nanomolar concentrations of nicotine into the preBötC increased respiratory frequency, whereas injection into the ipsilateral hypoglossal (XII) nucleus induced tonic/seizure-like activity. The $\alpha 4^{*}$-selective nAChR antagonist dihydro- $\beta$-erythroidine produced opposite effects and blocked the nicotinic responses. These data, showing that nAChRs in the preBötC and XII nucleus in L9'A mice are hypersensitive to nicotine and endogenous ACh, suggest that functional $\alpha 4^{\star} \mathrm{nAChRs}$ are present in the preBötC. They mediate cholinergic/nicotinic modulation of the excitability of preBötC inspiratory neurons and of respiratory rhythm. Furthermore, functional $\alpha 4^{\star} \mathrm{nAChRs}$ are present in XII nucleus and mediate cholinergic/nicotinic modulation of tonic activity in XIIn.
\end{abstract}

Key words: L9'A knock-in mice; $\alpha 4$ subunit; preBötzinger complex; inspiratory neurons; hypoglossal nucleus; nicotine

\section{Introduction}

Cholinergic modulation of the neuronal excitability in the preBötzinger complex (preBötC) affects respiratory rhythm and is mediated by both muscarinic acetylcholine receptors (AChRs) and nicotinic AChRs (nAChRs) (Shao and Feldman, 2000, 2001; Bellingham and Ireland, 2002). The nAChRs are of particular interest because they also mediate the effects of exogenously administered nicotine, e.g., via cigarette smoke. Activation of $n A C h R s$ in the preBötC affects the excitability of preBötC inspiratory neurons [a subset of these neurons is hypothesized to generate respiratory rhythm (Gray et al., 1999; Feldman et al., 2003)] and perturbs respiratory rhythm (Shao and Feldman, 2001, 2005). Impairment of the excitability of respiratory neurons and/or neurotransmission in the preBötC may underlie pathophysiology of disorders related to central control of breathing such as sudden infant death syndrome, whose incidence is strongly correlated with maternal smoking (Kinney et al., 1995; Anderson et al., 2005; Mitchell and Milerad, 2006).

Received May 11, 2007; revised Dec. 4, 2007; accepted Dec. 4, 2007.

This work was supported by Tobacco-Related Disease Research Program (California) Grants 10RT-0241 and 12RT-0245, by National Institutes of Health Grants HL 40959 and DA17279, and by Philip Morris USA/International. Correspondence should be addressed to Xuesi M. Shao, Department of Neurobiology, David Geffen School of Medicine at University of California, Los Angeles, Box 951763, Los Angeles, CA 90095-1763. E-mail: mshao@ucla.edu.

D0I:10.1523/JNEUROSCI.3666-07.2008

Copyright $\odot 2008$ Society for Neuroscience $\quad 0270-6474 / 08 / 280519-10 \$ 15.00 / 0$ $\alpha 4, \alpha 7$, and $\beta 2 \mathrm{nAChR}$ subunits are present in the rostral ventrolateral medulla, probably including the preBötC (Wada et al., 1989; Dominguez del Toro et al., 1994; Dehkordi et al., 2004). Most $\alpha 4$-containing $\left(\alpha 4^{*}\right)$ receptors in the mouse brain also contain the $\beta 2$ subunit $\left(\beta 2^{*}\right)$, and vice-versa (Whiteaker et al., 2006). Pharmacological studies suggest that the nAChRs in the preBötC mediating nicotinic modulation of respiratory pattern are of $\alpha 4 \beta 2^{\star}$ subtypes (Shao and Feldman, 2002). However, pharmacological tools to identify the specific molecular compositions of native nAChRs are limited. Genetically engineered nAChR subunit knock-out and knock-in animals provide a powerful approach to determine the subunit compositions of nAChRs and their functional roles in CNS (Champtiaux and Changeux, 2004). Knock-in mouse strains with a point mutation at the Leu9' position in the M2 pore-lining region of nAChR $\alpha 4$ subunits were developed. The $\alpha 4 \mathrm{~L} 9^{\prime} \mathrm{A}$ mouse strain was designed and selected to display largely normal physiology and behavior in the absence of added drugs. The strain resembles wild-type with regard to locomotion and ambulation, learning behavior, and anxiety; their neuroanatomy is normal. There are subtle differences from wild-type in their sleep-wake cycle. However, the L9'A mice are strikingly hypersensitive to several effects of nicotine: reward behavior, hypothermia, tolerance to hypothermia, locomotor sensitization, Straub tail, and seizures. This hypersensitivity to agonists is also displayed at the cellular and membrane levels in several preparations (Tapper et al., 2004; Fonck et al., 2005). 
These gain-of-function knock-in mouse strains allow tests of the hypothesis that activation of $\alpha 4^{\star}$ receptors is sufficient to produce a given response (Labarca et al., 2001; Fonck et al., 2003, 2005; Tapper et al., 2004, 2007). Using the L9'A mouse strain, we tested the hypothesis that activation of $\alpha 4^{*}$ nAChRs alters the excitability of neurons in the preBötC rhythmogenic network and that these nAChRs can mediate cholinergic/nicotinic modulation of respiratory rhythm. Identifying the molecular composition of nAChRs mediating the cholinergic modulation of respiratory pattern provides molecular basis for developing therapeutic agents targeting nAChRs (Picciotto et al., 2001; Gotti et al., 2006), both protective and interventional, with fewer side effects because of increased specificity.

\section{Materials and Methods}

$L 9^{\prime}$ A knock-in mice. These experiments used mice of the L9'A knock-in strain, backcrossed at least 10 generations to C57BL/6. Generation of the L9'A knock-in mice was reported in detail by Tapper et al. (2004) and Fonck et al. (2005). Only homozygous mutant knock-in mice and wildtype C57BL/6 mice were used in this study. The genotype of the mice used in the electrophysiological experiments was confirmed by PCR analysis of tail DNA.

Slice preparation. Experiments were performed on a medullary slice preparation that retains functional respiratory networks and generates respiratory rhythm in vitro (Smith et al., 1991). A neonatal mouse [postnatal day 0 (P0)-P3], either wild-type C57BL/6 or L9' A, was anesthetized with isoflurane and then promptly decerebrated. Tissue dissection and slicing were performed in an artificial CSF (ACSF) containing (in mM) $128 \mathrm{NaCl}, 3.0 \mathrm{KCl}, 1.5 \mathrm{CaCl}_{2}, 1.0 \mathrm{MgSO}_{4}, 23.5 \mathrm{NaHCO}_{3}, 0.5 \mathrm{NaH}_{2} \mathrm{PO}_{4}$, and 30 glucose, and equilibrated with $95 \% \mathrm{O}_{2}-5 \% \mathrm{CO}_{2}(\mathrm{pH}=7.4)$ at room temperature. The cerebellum was removed and the brainstemspinal cord was isolated. The brainstem-spinal cord was mounted in the specimen vise of a Vibratome (VT 100, Technical Products International, St. Louis, MO) oriented vertically with rostral end upward. The brainstem was sectioned serially in the coronal plane with a dissection microscope until the landmarks, e.g., nucleus ambiguus and inferior olive, at the rostral boundary of the preBötC were visible. One transverse slice (450-550 $\mu \mathrm{m}$ thick) was cut. The slice was transferred to a recording chamber of $1.2 \mathrm{ml}$ volume and stabilized with a threaded frame. During electrophysiological recordings, the slice was continuously perfused $(2 \pm$ $0.5 \mathrm{ml} / \mathrm{min}$ ) with ACSF with increased $\mathrm{KCl}(9 \mathrm{~mm})$ equilibrated with $95 \%$ $\mathrm{O}_{2}-5 \% \mathrm{CO}_{2}$. The ACSF in the recording chamber was maintained at $27 \pm 1^{\circ} \mathrm{C}$. All slices studied spontaneously generated respiratory rhythm at these conditions and the respiratory-related rhythmic activity can be recorded from the XIIn (Smith et al., 1991).

L9'A mutation expressed in Xenopus oocytes. Wild-type rat $\alpha 4$ and $\beta 2$ nicotinic subunits were subcloned into the pAMV vector. The Quikchange kit (Stratagene, La Jolla, CA) was used to construct the $\alpha 4 \mathrm{L9}^{\prime} \mathrm{A}$ mutations, which were verified by DNA sequencing. Capped cRNA was synthesized from linearized cDNA using the mMessage mMachine RNA transcription kit (Ambion, Austin, TX).

Stage V-VI Xenopus oocytes were surgically isolated, and surgeries performed, using methods approved by the Caltech Institutional Animal Care Committee. Isolated oocytes were injected with $4 \mathrm{ng}$ of mutated $\alpha 4$ and $4 \mathrm{ng}$ of wild-type $\beta 2 \mathrm{cRNA}$ in $50 \mathrm{nl}$ of $\mathrm{H}_{2} \mathrm{O}$. Before recording, injected oocytes were incubated for $2 \mathrm{~d}$ in a solution containing (in $\mathrm{mM}$ ) 96 $\mathrm{NaCl}, 5 \mathrm{HEPES}, 2.5 \mathrm{Na}$ pyruvate, $2 \mathrm{KCl}, 1.8 \mathrm{CaCl}_{2}, 1 \mathrm{MgCl}_{2}, 2.5 \mu \mathrm{g} / \mathrm{ml}$ gentamycin, and $5 \%$ horse serum, $\mathrm{pH} 7.4$, at $18^{\circ} \mathrm{C}$.

Electrophysiological recording. Neurons within $100 \mu \mathrm{m}$ of the slice surface were visualized with an infrared-differential interference contrast microscope (Axioskop2, Carl Zeiss, Göttingen, Germany). The respiratory neurons we recorded fired in phase with the inspiratory bursts of the XIIn rhythmic motor output and were located ventral to the nucleus ambiguus in the preBötC. Patch pipettes were pulled from thick wall $(0.32 \mathrm{~mm}$ ) borosilicate glass with tip size 1-1.5 $\mu \mathrm{m}$ (resistance: 4-6.5 $\mathrm{M} \Omega$ ). The pipette filling solution contained (in mM) $135 \mathrm{~K}$-gluconate, 5.0 $\mathrm{NaCl}, 0.1 \mathrm{CaCl}_{2}, 1.1$ EGTA, 10 HEPES, 2.0 ATP $\left(\mathrm{Mg}^{2+}\right.$ salt $)$, and 0.3 GTP
$\left(\mathrm{Na}^{+}\right.$salt), $\mathrm{pH}$ adjusted to 7.25 with $\mathrm{KOH}$. To maintain stable electrode potentials during whole-cell patch-clamp recordings, a micro-agar salt bridge of $3 \mathrm{M} \mathrm{KCl}$ was built in the electrode holder that formed an electrical connection between the pipette solution and the $\mathrm{Ag} / \mathrm{AgCl}$ wire connected to the headstage of a patch-clamp amplifier (Shao and Feldman, 2007a). Intracellular signals were amplified and low-pass filtered at $2 \mathrm{kHz}$ with a patch-clamp amplifier (MultiClamp 700B, Molecular Devices, Sunnyvale, CA). A $-10 \mathrm{mV}$ junction potential was determined experimentally; reported potential values are corrected for junction potential. For experiments in which drugs were bath applied, only one neuron was recorded from each preparation to avoid confounding effects of drug residue.

Respiratory-related rhythmic motor activity was recorded from the cut ends of XIIn with a suction electrode, amplified $(20,000 \times)$, and bandpass filtered ( $1 \mathrm{~Hz}$ to $3 \mathrm{kHz}$ ) with an amplifier (P5 series, Grass Instruments, Quincy, MA). Signals from intracellular recordings and from XIIn were digitized at $10 \mathrm{kHz}$ sampling frequency with the Digidata $1440 \mathrm{~A}$ and software CLAMPEX 10 (Molecular Devices) on a Pentiumbased computer. The two channels of signals were saved as data files for further analyses off-line.

We used an automated parallel two-electrode voltage-clamp (OpusXpress, Molecular Devices) to measure the agonist-induced currents in injected oocytes at $-60 \mathrm{mV}$. During recordings, oocytes were continually superfused with a nominally $\mathrm{Ca}^{2+}$-free saline (ND96) containing the following (in mM): $96 \mathrm{NaCl}, 5 \mathrm{HEPES}$, and $1 \mathrm{MgCl}_{2}, \mathrm{pH} 7.4$, to prevent activation of the endogenous $\mathrm{Ca}^{2+}$-activated $\mathrm{Cl}^{-}$current. Test solutions were applied at rate of $4 \mathrm{ml} / \mathrm{s}$ (chamber volume, $\sim 0.5 \mathrm{ml}$ ).

Drug application. Drugs were applied in two different ways: (1) bath application, i.e., adding them to the perfusate. In this procedure, stable recordings from a respiratory neuron and from XIIn can be obtained, and the drug concentrations for the slice are known accurately (drugs take effect in 2-4 min after adding them to the perfusate in our perfusion system); (2) pressure microinjection into the preBötC or XII nucleus. In this procedure, the location of drug action can be precisely identified. We estimate 1:10 reduction on average in drug concentration from the injection pipette to the target region (Liu et al., 1990). For microinjection experiments, a $5 \mu \mathrm{l}$ calibrated glass pipette (Drummond Scientific, Broomall, PA) was pulled, and the tip was broken to $6-9 \mu \mathrm{m}$ in diameter. The back of the pipette was connected to an air pressure source of 55-85 $\mathrm{kPa}$. The pipette was mounted on a micromanipulator and advanced into the preBötC or XII nucleus, $100-200 \mu \mathrm{m}$ below the slice surface. The injection volume was monitored by the displacement of the fluid meniscus using a microscope with a calibrated eyepiece reticule. A volume of 10 $\mathrm{nl}$ was injected, which is estimated to diffuse to a region approximating the size of the preBötC within $10 \mathrm{~s}$ (Nicholson, 1985). For injections into XII nucleus, 15-20 nl was injected. Drugs were dissolved in a pipette solution containing (in mM) $150 \mathrm{NaCl}, 9 \mathrm{KCl}, 1.5 \mathrm{CaCl}_{2}, 1.0 \mathrm{MgSO}_{4}, 10$ HEPES, and 30 glucose, $\mathrm{pH}$ adjusted to 7.4 with $\mathrm{NaOH}$.

For bath application of cholinergic drugs, the effects were measured 3-5 min after addition. The recordings of the neuronal and XIIn activity immediately before drug application served as controls for each preparation.

$(-)$-Nicotine (hydrogen tartrate salt) and dihydro- $\beta$-erythroidine hydrobromide (DH $\beta$ E) were obtained from Sigma (St. Louis, MO).

Data analysis. The respiratory-related motor activity recorded from XIIn was digitally integrated by full-wave rectification and low-pass filtering (digital RC filter) at a time constant of $40 \mathrm{~ms}$ using a data analysis software DataView V4.7 (W. J. Heitler, University of St. Andrews, St. Andrews, UK). The intracellular signals were digitally low-pass filtered at $1150 \mathrm{~Hz}$ (eight-pole Bessel filter) except for measuring the phasic inward current of inspiratory neurons, for which the intracellular signal was filtered at $20 \mathrm{~Hz}$. Respiratory periods were averaged from 10 consecutive periods in control or drug application conditions for each preparation, except in the microinjection experiments, in which 7-10 consecutive periods were averaged, as the nicotine effects were briefer after microinjection. Because the distribution of periods is Gaussian, periods were averaged across preparations for statistical tests. Respiratory frequency was taken as reciprocal of period and presented as mean \pm SD in the text. The amplitude (and duration) of integrated inspiratory bursts of XIIn 
A

\section{Wild-type}

$5 \mu \mathrm{M}$ nicotine

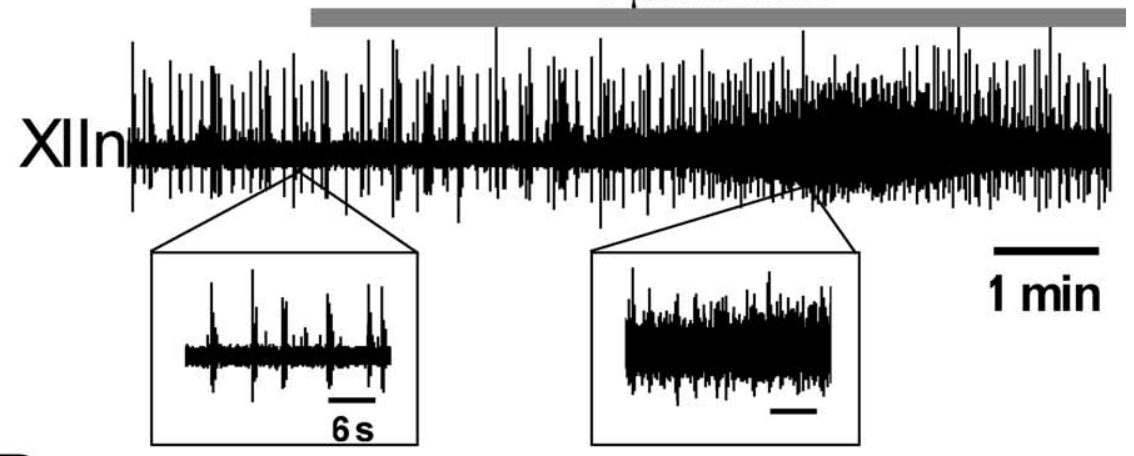

L9'A Knock-in $10 \mathrm{nM}$ nicotine

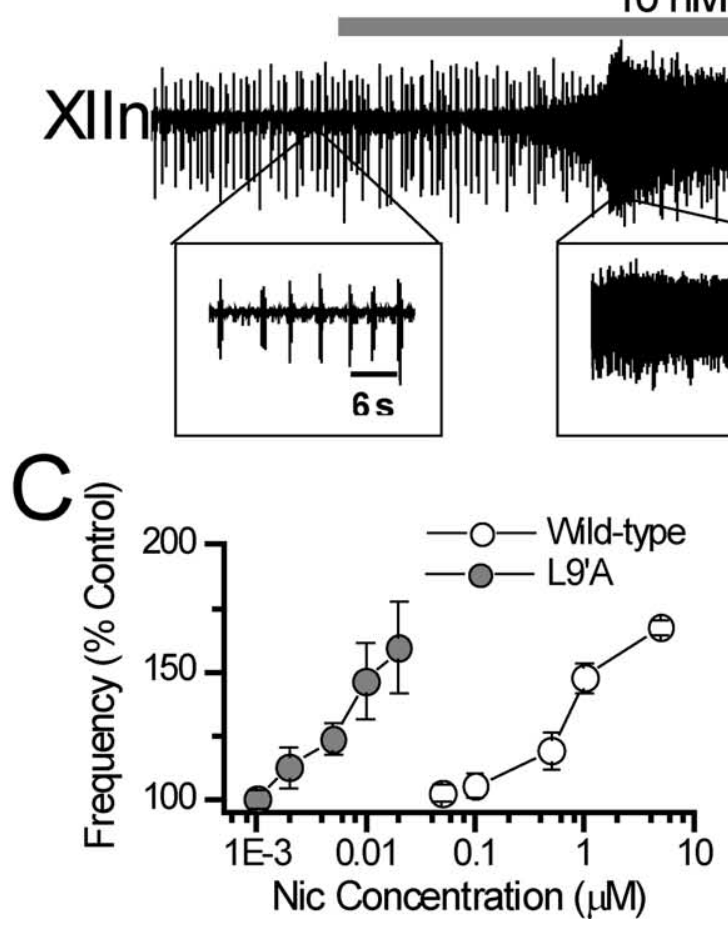

Figure 1. Nicotine (Nic) at low nanomolar concentrations increased frequency of respiratory rhythm and induced intense tonic/seizure-like activity in $L 9^{\prime} \mathrm{A}$ mice compared with effects of Nic at micromolar concentrations in wild-type mice in vitro. $\boldsymbol{A}$, Respiratory-related rhythmic activity recorded from the hypoglossal nerve roots (XIIn) in the medullary slice preparation of a wild-type mouse in response to bath application of $5 \mu \mathrm{m}$ Nic. Insets, Activities on an expanded time scale. $\boldsymbol{B}$, Respiratory-related rhythmic activity from a slice of $\mathrm{nAChR} \alpha 4$ subunit $\mathrm{L}^{\prime} \mathrm{A}$ knock-in mouse in response to bath application of $10 \mathrm{~nm}$ Nic. Time scales are equivalent for $\boldsymbol{A}$ and $\boldsymbol{B}$. Time scales for all insets are equivalent. $\boldsymbol{C}$, Concentration dependence of respiratory frequency in $\mathrm{L}^{\prime} \mathrm{A}$ and wild-type mice. Percentage (\%) control of frequency $=100 \times$ (frequency at the test Nic concentration)/(frequency in pre-Nic conditions). Data were averaged across preparations $(n=3-12)$ and presented as mean $\pm \mathrm{SE}$.

experiments with the antagonist $\mathrm{DH} \beta \mathrm{E}$ followed by nicotine, we measured electrophysiological parameters before and during application of $\mathrm{DH} \beta \mathrm{E}$ and during nicotine $+\mathrm{DH} \beta \mathrm{E}$ for each neuron in each slice. One-way repeatedmeasures ANOVA was used to test the statistical significance for drug treatments. Post hoc comparison analyses based on Tukey were used to determine the significance of differences between $\mathrm{DH} \beta \mathrm{E}$ application and control and between nicotine $+\mathrm{DH} \beta \mathrm{E}$ and $\mathrm{DH} \beta \mathrm{E}$ alone. The procedure MIXED in the data analysis software package SAS (V9, SAS Institute, Cary, NC) was used for these analyses. In all analyses, $p \leq 0.05$ was the criterion for statistical significance.

Membrane noise (current fluctuation of voltage-clamped inspiratory neurons) and spontaneous EPSCs (sEPSCs) during expiratory periods were analyzed with DataView 4.7. Membrane noise was quantified by calculating root-mean-square ( $\mathrm{rms}$, statistically equivalent to SD calculation) of the current recordings (inspiratory periods were excluded) at a bandwidth of 1-1000 Hz. Recording sections of 1-2 min that contained 200-2000 sEPSCs were taken from predrug control and drug application conditions. The program detected sEPSCs during expiratory periods by an optimally scaled template recognition algorithm (Clements and Bekkers, 1997). Rate, i.e., frequency of sEPSCs was determined by dividing the number of sEPSCs by total expiratory duration (inspiratory periods were subtracted) in the 1-2 min sections from predrug and drug application conditions. Then they were averaged across neurons. Differences in mean rates were tested with paired $t$ test or repeated-measures ANOVA as described above. sEPSC amplitude was analyzed with one-way repeated-measures ANOVA taking every sEPSC in the $1-2 \mathrm{~min} \mathrm{sec-}$ tions from predrug and drug application conditions recorded from an individual neuron as repeated measurements.

\section{Results}

Respiratory rhythm of $\mathrm{L9}^{\prime} \mathrm{A}$ mice is hypersensitive to nicotine at low nanomolar concentrations in vitro Respiratory-related rhythmic activity was recorded from XIIn in in vitro medullary slice preparations of wild-type and L9' A mice. In slices from L9'A mice, the frequency of rhythmic activity increased in response to bath application of nanomolar concentrations of nicotine, in contrast to the micromolar concentrations required to elicit responses in slices from wild-type mice (Fig. $1 A, B$ ). The nicotine concentra-

and the inspiratory drive current of inspiratory neurons were measured from the averaged envelope of five to six consecutive inspiratory periods triggered by the up stroke of the integrated inspiratory XIIn bursts (DataView 4.7). Durations were measured at $20 \%$ of peak amplitude. Then, they were averaged across neurons or preparations and presented as mean $\pm \mathrm{SD}$, except when indicated as mean $\pm \mathrm{SE}$ in the figures. $n=$ number of cells (for whole cell recording) or preparations (for XIIn motor output recording) is indicated in the text. For experiments with application of nicotine, paired $t$ tests (except as indicated otherwise) for significance were applied taking the measurements before (predrug control) and during nicotine application as a pair for each preparation. For tion-frequency response curve in $\mathrm{L}^{\prime} \mathrm{A}$ mice was greatly left shifted compared with that of wild type. Equipotent effects of nicotine occurred at concentrations $\sim 100$-fold lower in L9'A than in wild-type slices (Fig. 1C), suggesting that the nAChRs in L9' A mice are hypersensitive to nicotine and that the nAChRs mediating the modulatory effects on the respiratory pattern contain $\alpha 4$ subunits. In slices from L9'A mice, low nanomolar concentrations of nicotine also induced tonic/seizure-like activity, an effect seen in wild-type mice only at micromolar nicotine (Fig. 
$1 A, B)$. The intense tonic/seizure-like activities vitiated systematic frequency measurements for increases more than $\sim 50 \%$; therefore, we did not calculate $\mathrm{EC}_{50}$ values. To insure inducing significant responses while avoiding intense seizure-like activity in the subsequent cellular experiments, we used $5 \mathrm{~nm}$ nicotine for L9' A preparations and $1 \mu \mathrm{M}$ nicotine for wild-type preparations.

In control conditions without nicotine application, the respiratory frequency of preparations from L9'A mice was higher than that from wild-type mice. Mean respiratory frequency of $\mathrm{L}^{\prime}$ 'A slice was $11.9 \pm 2.9$ inspiratory bursts/min versus $9.8 \pm 3.2 / \mathrm{min}$ in wild-type $(p=0.018$, nonpaired $t$ test, $n=33$ vs $n=29)$. This result suggests that the hypersensitive L9'A nAChRs are tonically responding to the endogenous ligands, such as ACh, spontaneously released from presynaptic structures in slices.

\section{Reponses of preBötC inspiratory} neurons of L9' A mice to nicotine at low nanomolar concentrations

To test the hypothesis that nicotine acting via $\alpha 4^{*}$ nAChRs depolarizes preBötC neurons producing increases in respiratory frequency, we recorded membrane potentials from whole-cell current-clamped preBötC inspiratory neurons in the slice preparations. These neurons discharge in synchrony with the respiratory-related rhythmic motor activity simultaneously recorded from XIIn. We applied hyperpolarizing current to maintain preBötC inspiratory neurons at a baseline membrane voltage of -70 to $-65 \mathrm{mV}$ in the absence of drug. Bath application of $5 \mathrm{~nm}$ nicotine to slices from L9' A mice depolarized these neurons and induced tonic spiking (Fig. 2). At the same time, nicotine increased the frequency of respiratoryrelated rhythmic activity and induced tonic activity, as shown by the elevated baseline between inspiratory bursts in the integrated XIIn traces ( $\int \mathrm{XIIn}, n=3$ ). The neuron shown in Figure 2 was a pacemaker neuron that fired ectopic bursts (indicated by arrows in the insets) during expiratory periods. In agreement with previous data (Shao and Feldman, 2002), we observed similar responses with a nicotine concentration of $1 \mu \mathrm{M}$ in wild-type slices (data not shown). Addition of $0.2 \mu \mathrm{M} \mathrm{DH} \beta \mathrm{E}$ reversed these effects; it hyperpolarized inspiratory neurons and produced a corresponding decrease in the respiratory frequency and suppressed the tonic activity in XIIn (Fig. 2). The fact that the frequency fell below the predrug control level with nicotine plus $\mathrm{DH} \beta \mathrm{E}$ suggests that there is endogenous $\mathrm{ACh}$ release in the preBötC acting on the hypersensitive $\alpha 4^{*} \mathrm{nAChRs}$ to modulate respiratory rhythm. $\mathrm{DH} \beta \mathrm{E}$ applied in the presence of nicotine blocked both the effects of nicotine and the effects of endogenous ACh on the knock-in $\alpha 4^{\star}$ nAChRs.

For more quantitative analysis of neuronal responses, we recorded from preBötC inspiratory neurons in voltage-clamp mode (holding potential $V_{\mathrm{h}}=-65 \mathrm{mV}$ ). With slices from wildtype mice, application of $1 \mu \mathrm{M}$ nicotine increased respiratory frequency from $9.2 \pm 3.0 / \mathrm{min}$ to $13.5 \pm 4.1 / \mathrm{min}(p<0.001, n=$ 12) without affecting the amplitude of integrated XIIn rhythmic activity (10.4 $\pm 12.9 \mu \mathrm{V}$ in control, i.e., prenicotine conditions vs $9.6 \pm 12.0 \mu \mathrm{V}$ in the presence of nicotine, $p=0.89, n=12$ ) (Fig. $3 A, B, E)$. In the voltage-clamped neurons, nicotine induced (1) a tonic inward current of $17.3 \pm 10.8 \mathrm{pA}$ (measured as baseline current during expiratory periods during nicotine application minus baseline current in prenicotine conditions; $p=0.005, n=$ 7) associated with an increase in membrane noise (current fluctuation) [rms value of the membrane noise increased from $4.47 \pm 1.37$ to $6.42 \pm 1.47 \mathrm{pA}(p=0.0084, n=5)($ Fig. $3 A, F)$. Membrane noise consists of extrasynaptic channel activity, including ligand-gated and voltage-gated channels, resolved and unresolved sEPSCs and sIPSCs, and random noise. A tonic inward current associated with an increase in membrane noise suggests predominantly excitatory extrasynaptic and/or synaptic channel activation.]; (2) a decrease in amplitude of phasic inspiratory drive current from $65.1 \pm 47.4$ to $56.3 \pm 44.0 \mathrm{pA}$ ( $p=$ $0.01, n=11)$ and duration from $447 \pm 111$ to $388 \pm 125 \mathrm{~ms}$ (measured at $20 \%$ of peak, $p=0.032$ ) (Fig. $3 B, F$ ); (3) an increase in frequency of sEPSCs during expiratory periods from $10.9 \pm$ $5.0 / \mathrm{s}$ to $14.7 \pm 6.0 / \mathrm{s}(p=0.012, n=6)$ and an increase in amplitude of sEPSCs from $-13.0 \pm 3.3$ to $-15.1 \pm 3.6 \mathrm{pA}(p=$ 0.0002 ) (Fig. $3 A$, insets, $F$ ). Figure $3, E$ and $F$, shows the summary data of XIIn activity and single neuron parameters respectively as percentage changes referred to prenicotine application. These responses slightly desensitized when nicotine was present for $>6$ min.

With L9'A slices, bath application of $5 \mathrm{~nm}$ nicotine increased respiratory frequency from $12.4 \pm 1.9 / \mathrm{min}$ to $15.3 \pm 3.1 / \mathrm{min}$ ( $p=0.002, n=9$ ) without affecting the amplitude of integrated XIIn rhythmic activity $(7.1 \pm 5.2 \mu \mathrm{V}$ in prenicotine control conditions vs $7.7 \pm 5.0 \mu \mathrm{V}$ in the presence of nicotine, $p=0.21, n=$ 8) (Fig. $3 C-E$ ). In the voltage-clamped neurons, $5 \mathrm{~nm}$ nicotine 


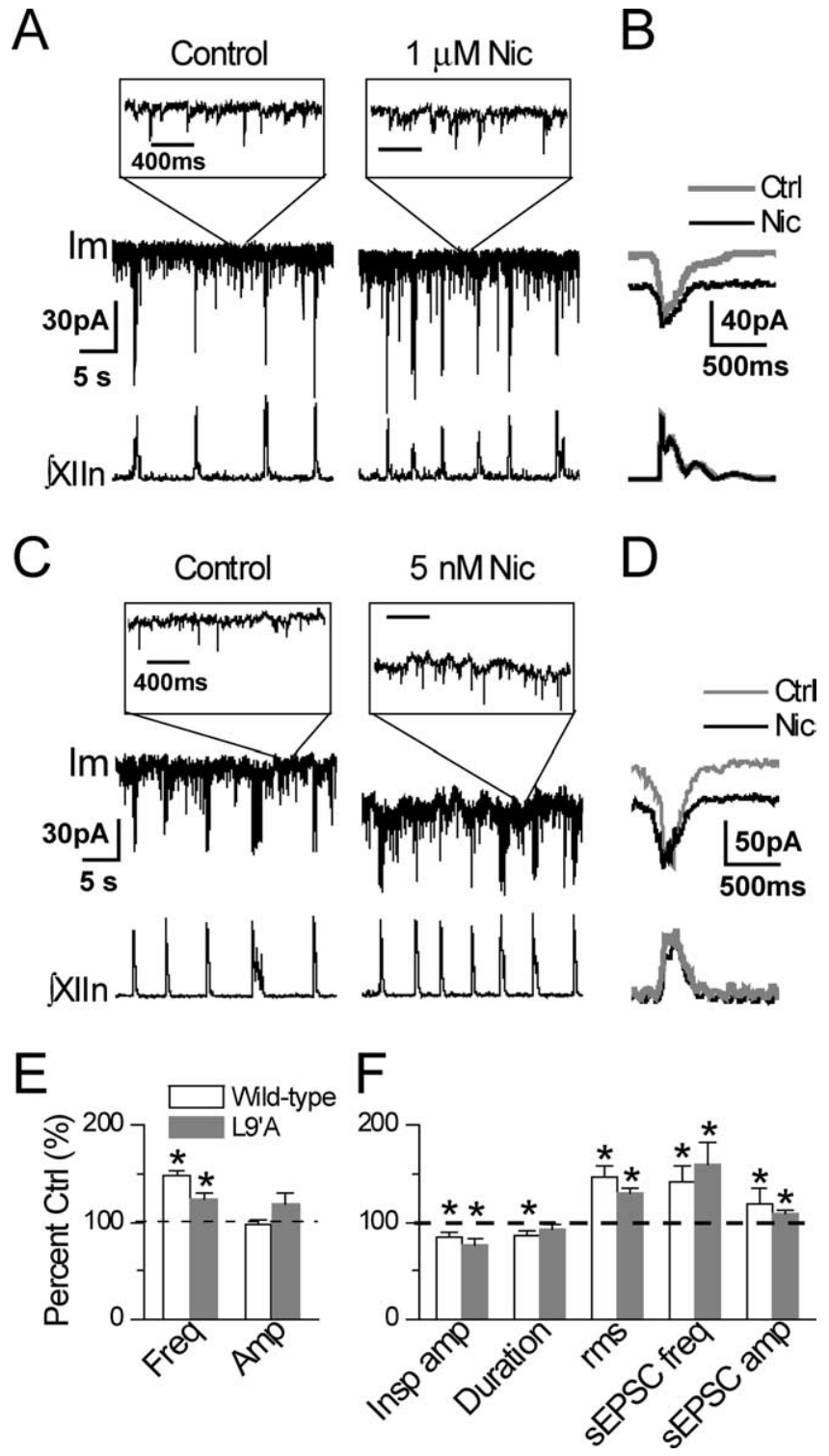

Figure 3. Responses of voltage-clamped preBötC inspiratory neurons and XIIn rhythmic activity to nicotine (Nic) application in L9'A and wild-type mice. A, A preBötC inspiratory neuron and the respiratory-related XIIn rhythmic motor activity in a slice preparation from a wild-type mouse in the absence (left) and presence (right) of bath-applied $1 \mu \mathrm{M} \mathrm{Nic.} I_{\mathrm{m}}$ is membrane current of the neuron (holding potential $V_{\mathrm{h}}=-65 \mathrm{mV}$ ). $\int X$ IIn, Integrated XIIn activity. Insets, $I_{\mathrm{m}}$ recordings on an expanded time scale at time sections indicated, revealing SEPSCS. Time scales for the two insets are equivalent. $y$-Scales for the continuous recordings and all the insets are equivalent. $\boldsymbol{B}$, Phasic inspiratory drive current of a preBötC inspiratory neuron. Averaged envelopes of five to six consecutive inspiratory periods for both $I_{m}$ and $\int$ XIIn channels in Nic application versus pre-Nic control (Ctrl) conditions. Im traces were low-pass filtered at $20 \mathrm{~Hz}$. Average was triggered by the up strokes of the integrated inspiratory bursts from XIIn. Duration of inspiratory drive current was measured at $20 \%$ of peak amplitude. Traces were from recordings of a different slice from those of $A$. $C$, As in $A$, a preBötC inspiratory neuron and the XIIn rhythmic activity in a slice preparation from an $\mathrm{L}^{\prime} \mathrm{A}$ mouse in the absence (left) and presence (right) of bath applied $5 \mathrm{~nm}$ Nic. $\boldsymbol{D}, \mathrm{As}$ in $\boldsymbol{B}$, phasic inspiratory drive current of a preBötC inspiratory neuron from an $\mathrm{L}^{\prime} \mathrm{A}$ slice. E, Summary data (mean \pm SE) of XIIn rhythmic activity for $\mathrm{L9}^{\prime} \mathrm{A}$ mice in response to $5 \mathrm{~nm}$ Nic versus wild-type mice in response to $1 \mu \mathrm{m}$ Nic. Freq, Frequency; Amp, amplitude. $\boldsymbol{F}$, Summary data of preBötC inspiratory neurons of $L^{\prime}{ }^{\prime}$ A mice in response to 5 nм Nic versus wild-type mice in response to $1 \mu \mathrm{m}$ Nic applications. Insp amp, Phasic inspiratory drive current amplitude; Duration, duration of inspiratory drive current; $r m s$, root-mean-square of membrane noise during expiratory periods; SEPSC freq, spontaneous EPSC frequency during expiratory periods: number of sEPSCs divided by total expiration time during 1-2 min recording time sections. Each section contained $200-2000$ sEPSCs. ${ }^{*} p \leq 0.05$ between Nic application and pre-Nic control. Numbers of neurons or preparations $(n)$ for each experiment are indicated in the text of Results. induced (1) a tonic inward current of $34.8 \pm 29.7 \mathrm{pA}$ ( $p=0.035$, $n=6$ ) associated with an increase in membrane noise (rms value from $5.62 \pm 2.11$ to $7.34 \pm 2.57 \mathrm{pA}, p=0.0046, n=5$ ) (Fig. $3 C, F)$; (2) a decrease in the amplitude of inward phasic inspiratory drive current from $103.2 \pm 149.3$ to $90.7 \pm 149.0 \mathrm{pA}(p=$ $0.049, n=6)$ without affecting the duration (421 $\pm 117 \mathrm{~ms}$ in control conditions vs $383 \pm 78 \mathrm{~ms}$ in the presence of nicotine, $p=0.28, n=5)$ (Fig. 3D,F); (3) an increase in the frequency of sEPSCs during expiratory periods from $10.5 \pm 5.5 / \mathrm{s}$ to $15.3 \pm$ $6.4 / \mathrm{s}(p=0.015, n=5)$ and an increase in amplitude of sEPSCs from $-17.7 \pm 4.9$ to $-19.3 \pm 5.4 \mathrm{pA}(p=0.0037)$ (Fig. $3 C$, insets, $F$ ). Figure $3, E$ and $F$, shows the summary data for XIIn activity and single neuron parameters respectively as percentage changes referred to prenicotine application as controls. These responses slightly desensitized when nicotine was present for $>6 \mathrm{~min}$.

These results show that the nAChRs mediating nicotinic effects on inspiratory neurons in the preBötC rhythmogenic network in L9'A mice are hypersensitive to nicotine; therefore, these nAChRs contain $\alpha 4$ subunits. Activation of $\alpha 4^{*}$ nAChRs facilitates excitatory glutamatergic input to these neurons and induces a tonic inward current that enhances the excitability of the neurons. Depolarization of these neurons results in increased respiratory frequency.

\section{DH $\beta E$ blocks the nicotine effects on preBötC inspiratory neurons and on respiratory rhythm and induces opposite effects in L9' A mice}

The effects of nicotine on preBötC inspiratory neurons and on the pattern of respiratory-related motor activity in neonatal rats can be blocked by DH $\beta$ E (Shao and Feldman, 2002), a selective antagonist of $\alpha 4^{\star}$ nAChRs, although not completely specific (Harvey and Luetje, 1996; Harvey et al., 1996). We tested whether this antagonist blocks the effects of low nanomolar concentrations of nicotine on preBötC inspiratory neurons and respiratory rhythm in L9' A slices by applying $\mathrm{DH} \beta \mathrm{E}$ before exposure to nicotine. Bath application of $\mathrm{DH} \beta \mathrm{E}(0.1-0.2 \mu \mathrm{M})$ decreased frequency from $10.2 \pm 3.0 / \mathrm{min}$ to $5.5 \pm 2.4 / \mathrm{min}(p=0.033, n=4)$ with no significant effect on the amplitude of integrated inspiratory bursts of XIIn motor output $(7.6 \pm 6.9 \mu \mathrm{V}$ in control conditions vs $8.0 \pm 7.5 \mu \mathrm{V}$ in the presence of $\mathrm{DH} \beta \mathrm{E}, p=0.65, n=4$ ) (Fig. $4 A-C$ ). In contrast to nicotine, $\mathrm{DH} \beta \mathrm{E}$ alone induced (1) a tonic outward current of $8.3 \pm 2.5 \mathrm{pA}(p=0.028, n=3)$ associated with a decrease in rms value of membrane noise from $6.26 \pm 2.89$ to $4.10 \pm 1.52 \mathrm{pA}(p=0.022, n=4)$ (Fig. $4 A, D)$; (2) a statistically insignificant increase in amplitude of phasic inspiratory drive current from $67.6 \pm 47.0$ to $74.8 \pm 52.2 \mathrm{pA}$ ( $p=$ $0.43)$ and in duration from $475 \pm 162 \mathrm{~ms}$ to $560 \pm 184 \mathrm{~ms}(p=$ $0.41, n=4)$ (Fig. $4 B, D$ ); (3) a decrease in frequency of sEPSCs during expiratory periods from $16.7 \pm 11.1 / \mathrm{s}$ to $8.7 \pm 4.5 / \mathrm{s}(p=$ 0.023 ) and in amplitude of sEPSCs from $-17.4 \pm 5.4$ to $-13.9 \pm$ $2.5 \mathrm{pA}(p<0.001, n=4)$ (Fig. $4 A, D)$. The results that application of $\mathrm{DH} \beta \mathrm{E}$ alone produced effects opposite to those of nicotine in L9' A slices suggest that there is endogenous ligand release, such as $\mathrm{ACh}$, in the preBötC, acting on the hypersensitive $\alpha 4^{*}$ nAChRs to modulate respiratory rhythm. Evidently the hypersensitive nAChRs are not completely desensitized by the background levels of endogenous $\mathrm{ACh}$, consistent with the finding that the hypersensitive receptors have a "window" of activation in the sustained presence of agonist (Fonck et al., 2005).

Subsequent addition of nicotine (5 nM) in the presence of $\mathrm{DH} \beta \mathrm{E}$ did not have further effect on respiratory rhythm and on most parameters measured in preBötC inspiratory neurons. Application of nicotine induced insignificant changes in respiratory 


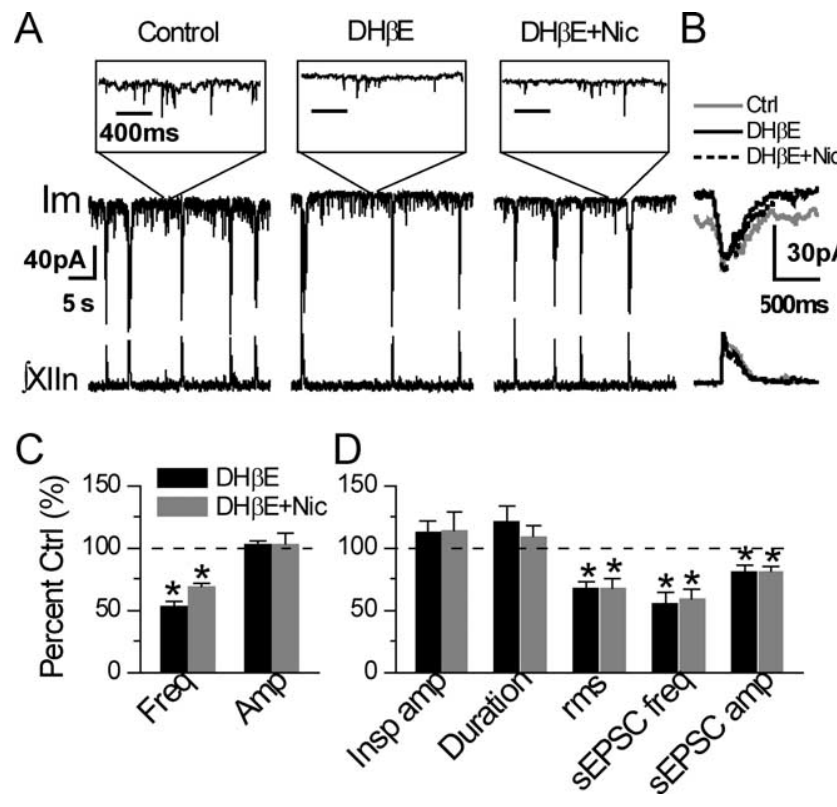

Figure 4. $\alpha 4^{*}$ selective nicotinic antagonist $\mathrm{DH} \beta$ E induced opposite effects and blocked the effects of nicotine (Nic) on preBötC inspiratory neurons and on the XIIn rhythmic activity in L9' $\mathrm{A}$ mice. $A$, preBötC inspiratory neuron and respiratory-related XIIn rhythmic activity in a $L^{\prime}$ A slice in response to bath application of $0.2 \mu \mathrm{m} \mathrm{DH} \beta \mathrm{E}$ followed by $5 \mathrm{~nm}$ Nic in the presence of $\mathrm{DH} \beta \mathrm{E}$ (DH $\beta \mathrm{E}+\mathrm{Nic}) . I_{\mathrm{m}}$, Membrane current of the neuron $\left(V_{\mathrm{h}}=-65 \mathrm{mV}\right)$. $\int \mathrm{XIIn}$, Integrated XIIn activity. Insets, $I_{m}$ recordings on an expanded time scale at time sections indicated to show SEPSCS. Time scales for the three insets are equivalent. $y$-Scales for the continuous recordings and the insets are equivalent. $\boldsymbol{B}$, Phasic inspiratory drive current of a preBötC inspiratory neuron from a different slice. Averaged envelopes of five to six consecutive inspiratory periods for both $I_{\mathrm{m}}$ and $\int X \mathrm{IIn}$ traces in the conditions of predrug control (Ctrl), DH $\beta$ E application, and $\mathrm{DH} \beta \mathrm{E}+$ $\mathrm{Nic} I_{\mathrm{m}}$ traces were low-pass filtered at $20 \mathrm{~Hz}$. Average was triggered by the up strokes of the integrated inspiratory bursts from XIIn. Durations of inspiratory drive current were measured at $20 \%$ of peak amplitude. C, Summary data (mean \pm SE) of XIIn rhythmic activity of $L 9^{\prime} A$ in response to $0.2 \mu \mathrm{m} \mathrm{DH} \beta \mathrm{E}$ application followed by $\mathrm{DH} \beta \mathrm{E}+\mathrm{Nic}$. Freq, Frequency; Amp, amplitude. $D$, Summary data of preBötC inspiratory neurons in response to $\mathrm{DH} \beta$ E followed by $\mathrm{DH} \beta \mathrm{E}$ + Nic. Insp amp, Phasic inspiratory drive current amplitude; Duration, duration of inspiratory drive current; rms, root-mean-square of membrane noise during expiratory periods; $s E P S C$ freq, spontaneous EPSC frequency during expiratory periods: number of SEPSCS divided by total expiration time during $1-2$ min recording times. ${ }^{*} p \leq 0.05$, statistical significance between drug application and predrug control.

frequency to $6.9 \pm 1.6 / \mathrm{min}(p=0.19, n=4)$ and in amplitude of inspiratory bursts to $8.0 \pm 7.8 \mu \mathrm{V}(p=0.998)$ (Fig. $4 A, C)$. In voltage-clamped inspiratory neurons, nicotine induced insignificant changes in tonic outward current to $9.8 \pm 9.2 \mathrm{pA}(p=0.78$, $n=3)$ and in membrane noise to $4.29 \pm 2.11 \mathrm{pA}(p=0.94, n=$ 4) (Fig. $4 A, D$ ), insignificant changes in amplitude of phasic inspiratory drive current to $75.4 \pm 53.8 \mathrm{pA}(p=0.99)$ and in duration to $507 \pm 154 \mathrm{~ms}(p=0.68, n=4)$ (Fig. $4 B, D)$, and insignificant changes in frequency of sEPSCs during expiratory periods to $9.9 \pm 6.5 / \mathrm{s}(p=0.68, n=4)$ and in amplitude of sEPSC to $-14.0 \pm 4.2(p=0.27)$ (Fig. $4 A, D)$.

These results show that the nicotine effects at nanomolar concentrations on preBötC inspiratory neurons and respiratory rhythm in $\mathrm{L}^{\prime} \mathrm{A}$ slices can be blocked by $\mathrm{DH} \beta \mathrm{E}$. These results are consistent with the hypothesis that $\alpha 4^{*}$ nAChRs mediate cholinergic/nicotinic modulation of the excitability of preBötC inspiratory neurons and regulation of respiratory rhythm.

\section{$\mathrm{DH} \beta \mathrm{E}$ blocks the responses of $\left(\alpha 4 \mathrm{~L}^{\prime} \mathrm{A}\right) \boldsymbol{\beta} 2 \mathrm{nAChRs}$ to nicotine and $\mathrm{ACh}$}

To test whether $\mathrm{DH} \beta \mathrm{E}$ remains a potent antagonist for $\mathrm{L9}^{\prime} \mathrm{A}$ mutated receptors, we expressed $\left(\alpha 4 \mathrm{~L} 9^{\prime} \mathrm{A}\right) \beta 2$ receptors in oo-

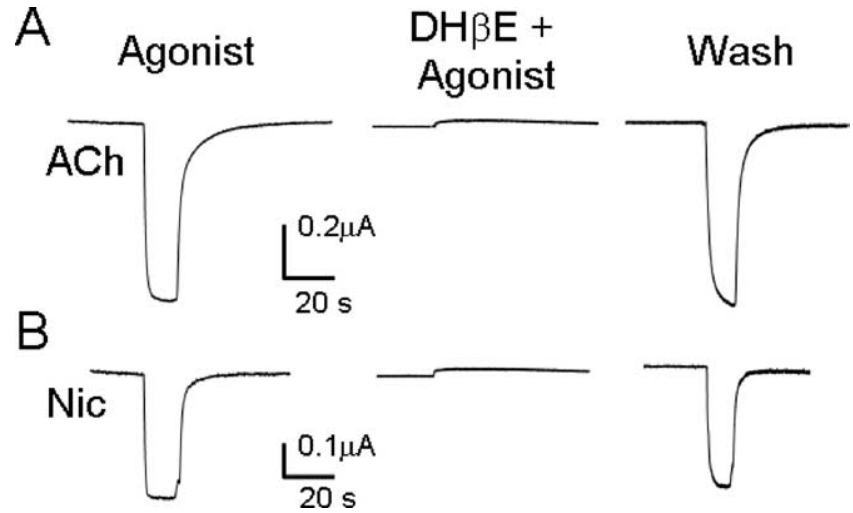

Figure 5. Reversible block of $\left(\alpha 4 L^{\prime} 9^{\prime} A\right) \beta 2$ receptors expressed in oocytes by $0.2 \mu \mathrm{m} \mathrm{DH} \beta \mathrm{E}$. $\boldsymbol{A}, \boldsymbol{B}$, Agonist concentration in each case was the $\mathrm{EC}_{20}$ for ACh $(0.125 \mu \mathrm{m}, \boldsymbol{A})$ or Nic $(0.025 \mu \mathrm{m}, \boldsymbol{B})$, applied for $15 \mathrm{~s}$. Left, first agonist application. Oocytes were washed with recording buffer for 2 $\mathrm{min}$ (not shown) and then perfused with $0.2 \mu \mathrm{M} \mathrm{DH} \beta \mathrm{E}$ for 5 min. Middle, The subsequent response to coapplied agonist $+0.2 \mu \mathrm{m} \mathrm{DH} \beta$ E. Oocytes were then washed with buffer for 5 $\mathrm{min}$, and an additional $15 \mathrm{~s}$ application of agonist was given (right). These data are typical of $n>5$ oocytes for each agonist. The small outward deflections are solution change artifacts.

cytes. Because $\mathrm{DH} \beta \mathrm{E}$ had modest effects on the rhythmic activity and sEPSC frequency in the slice preparation (Fig. 4), we inferred that $\mathrm{DH} \beta \mathrm{E}$ blocked the effect of endogenous $\mathrm{ACh}$, presumably at concentrations that were well below saturating levels. We therefore tested agonists (ACh or nicotine) at their $\mathrm{EC}_{20}(0.125$ and $0.025 \mu \mathrm{M}$, respectively, verified during the present experiments) (Fonck et al., 2005). The agonist-induced currents were completely and reversibly blocked by $0.2 \mu \mathrm{M} \mathrm{DH} \beta \mathrm{E}$ (Fig. 5). These results suggest that $\mathrm{DH} \beta \mathrm{E}$ is still a potent blocker for the hypersensitive $\alpha 4 \mathrm{~L} 9^{\prime} \mathrm{A}$ nAChRs (as it is for wild-type $\alpha 4 \beta 2$ receptors). These results are also consistent with a previous oocyte expression study: $\mathrm{DH} \beta \mathrm{E}$ remains a potent blocker when $\alpha 4$ Leu9' Thr, an even more hypersensitive mutation than the present L9'A mutation, is incorporated into the $\alpha 4$ subunit of $\alpha 4 \beta 2 \mathrm{nAChRs}$ (Moroni et al., 2006).

$\alpha 4^{\star} \mathrm{nAChRs}$ in the preBötC mediate nicotinic modulation of respiratory rhythm, and these receptors in the XII nucleus mediate nicotinic effects on the tonic activity

In slice preparations, we can pressure inject determined volumes of drugs into precisely located regions. To test whether the pre$\mathrm{BötC}$ is responsible for the nicotinic modulation of respiratory rhythm via $\alpha 4^{*} \mathrm{nAChRs}$, we microinjected nicotine at appropriately low concentrations locally into the preBötC in L9'A slices and recorded the respiratory-related rhythmic motor activity from XIIn. For comparison, we also injected nicotine into the XII nucleus.

Unilateral microinjection of $50 \mathrm{~nm}$ nicotine (10-15 nl; refer to Materials and Methods section) increased respiratory frequency from $9.8 \pm 1.8 / \mathrm{min}$ to $14.2 \pm 2.9 / \mathrm{min}(p<0.001, n=9)$ (Fig. $6 A)$. These microinjections did not affect the amplitude (15.0 \pm $5.5 \mu \mathrm{V}$ in prenicotine control conditions vs $13.9 \pm 4.6 \mu \mathrm{V}$ after nicotine injection, $p=0.07, n=9$ ) of integrated inspiratory bursts of XIIn motor output (Fig. $6 A, B$ ). Similar effects on frequency were observed after either ipsilateral or contralateral microinjection of nicotine. Bath application of $0.1-0.5 \mu \mathrm{M} \mathrm{DH} \beta \mathrm{E}$ greatly reduced the frequency effect induced by nicotine injection into the preBötC $(8.6 \pm 1.9 / \mathrm{min}$ in prenicotine conditions vs $10.1 \pm 1.2 / \mathrm{min}$ after nicotine injection; $p=0.074, n=6$ ). Nicotine injection did not affect the peak amplitude of integrated XIIn rhythmic motor activity $(11.8 \pm 6.8 \mu \mathrm{V}$ in prenicotine condi- 
A
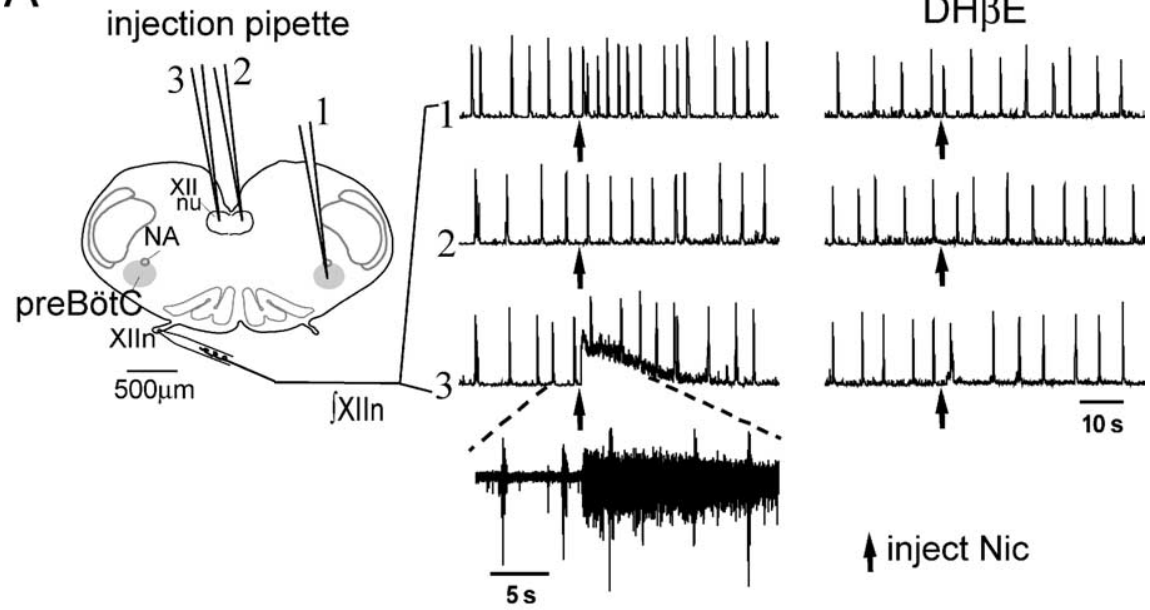

$\uparrow$ inject Nic
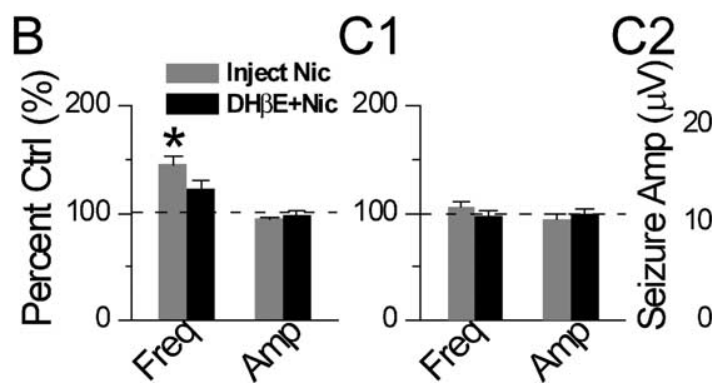

Figure 6. Microinjection of low nanomolar concentrations of nicotine (Nic) into the preBötC increased the frequency of respiratory-related XIIn rhythmic activity, and into hypoglossal nucleus (XII nu) induced tonic/seizure-like activity in L9'A mice. $\mathrm{DH} \beta \mathrm{E}$ greatly reduced these effects. $A$, Microinjection locations, the responses to Nic injections recorded from XIIn in the absence (middle) and presence (right) of bath-applied DH $\beta E(0.1-0.5 \mu \mathrm{M})$. Trace 1, Injection of $50 \mathrm{~nm} \mathrm{Nic} \mathrm{(10-15} \mathrm{nl}$ ) into contralateral preBötC corresponding to the injection pipette\#1. Trace 2, Injection of Nic (15-20 nl) into contralateral XII nu corresponding to the injection pipette \#2. Trace 3, Injection of Nic (15-20 nl) into ipsilateral XII nu corresponding to the injection pipette \#3. SXIIn, Integrated XIIn activity. Inset, Raw (nonintegrated) XII activity on an expanded time scale from the indicated section of trace 3 showing seizure-like activity. Injection pipettes were inserted into the target areas $100-200 \mu \mathrm{m}$ below the surface of the slice. Arrow indicates the time of Nic injection. $\boldsymbol{B}$, Summary data (mean \pm SE) of frequency (Freq) and amplitude (Amp) of integrated XIIn rhythmic activity in response to Nic injection into the preBötC in the absence and presence of bath applied $\mathrm{DH} \beta \mathrm{E}$. Amplitude was determined with averaged envelopes of five to six consecutive inspiratory bursts for each condition. C, Summary data of the effects of Nic injection into ipsilateral XII nu in the absence and presence of bath-applied DH $\beta E$ on frequency and amplitude of integrated XIIn rhythmic activity (C1), Nic-induced tonic/seizure-like activity amplitude (Seizure Amp; (2), and duration (Seizure Dur; (3) measured at $20 \%$ of peak amplitude of integrated XIIn activity. * Statistically significant difference between Nic injection versus pre-Nic conditions (paired $t$ test). ${ }^{\triangle}$ Statistically significant difference of Nic effects between the absence and presence of bath applied DH $\beta$ E.

tions vs $11.3 \pm 5.4 \mu \mathrm{V}$ after nicotine injection; $p=0.47, n=6)$ in the presence of $\mathrm{DH} \beta \mathrm{E}$ (Fig. $6 A, B$ ).

Microinjection of $50 \mathrm{~nm}$ nicotine (15-20 nl) into the ipsilateral XII nucleus induced tonic/seizure-like activity (Fig. $6 \mathrm{~A}$, trace 3) that had a peak amplitude of $12.0 \pm 9.4 \mu \mathrm{V}$ (integrated nerve recording) and lasted $41.1 \pm 21.0 \mathrm{~s}$ (measured by decline to $20 \%$ of the peak amplitude; $n=7$ ) (Fig. 6C2,C3). During these nicotine-induced seizures, the rhythmic inspiratory activity continued at near-control frequency $(10.7 \pm 1.6 / \mathrm{min}$ in prenicotine control conditions vs $11.1 \pm 0.9 / \mathrm{min}$ after nicotine injection, $p=$ $0.26, n=7)$ and amplitude $(13.7 \pm 6.6 \mu \mathrm{V}$ vs $12.9 \pm 6.3 \mu \mathrm{V}, p=$ $0.28)$ (Fig. $6 A, C 1$ ). There was no effect when nicotine was injected into the contralateral XII nucleus (Fig. 6A, trace 2); this served as a control for nonspecific mechanical effects of injections. Bath application of $\mathrm{DH} \beta \mathrm{E}(0.1-0.5 \mu \mathrm{M})$ greatly reduced the amplitude and duration of the tonic/seizure-like activity induced by microinjection of nicotine into the ipsilateral XII nucleus to $2.9 \pm 2.3 \mu \mathrm{V}(p=0.028$, paired $t$ test, $n=7)$ and $6.8 \pm$
4.7 s $(p=0.002)$, respectively (Fig. $6 C 2, C 3)$. In the presence of $\mathrm{DH} \beta \mathrm{E}$, nicotine injection into the ipsilateral XII nucleus has no effect on frequency $(7.6 \pm$ $2.2 / \mathrm{min}$ in prenicotine conditions vs $7.3 \pm$ $1.8 / \mathrm{min}$ after nicotine injection, $p=0.89$, $n=7)$ or on amplitude $(11.9 \pm 6.4 \mu \mathrm{V}$ vs $11.8 \pm 6.1 \mu \mathrm{V}, p=0.97)$ of the integrated XIIn rhythmic activity (Fig. 6C1).

These results suggest that functional $\alpha 4^{\star}$ nAChRs are present both in the preBötC and the XII nucleus. However, the effects of activating these receptors differ: $\alpha 4^{*} \mathrm{nAChRs}$ in the preBötC mediate $\mathrm{ACh} /$ nicotinic modulation of respiratory frequency, and $\alpha 4^{\star}$ nAChRs in the XII nucleus mediate $\mathrm{ACh} /$ nicotine induced tonic/seizure-like activity in XIIn.

\section{Discussion}

This study demonstrates several points related to nicotinic receptor composition and its function in the neural control of respiration. (1) The concentration-response relationship of respiratory rhythm in L9'A mice was greatly shifted to lower nicotine concentrations compared with that in wild-type mice in vitro. Respiratory frequency in predrug control conditions in L9'A mice was higher than that of wildtype mice, suggesting that the respiratory rhythm of L9'A mice is hypersensitive to nicotine and endogenous ACh. (2) Responses induced by $5 \mathrm{~nm}$ nicotine in preBötC inspiratory neurons and respiratoryrelated XIIn rhythmic activity in L9'A mice resembled $1 \mu \mathrm{M}$ nicotine-induced responses in wild-type mice. These responses included (i) depolarization of current-clamped inspiratory neurons; (ii) a tonic inward current in voltage-clamped inspiratory neurons associated with an increase in membrane noise; (iii) a decrease in phasic inspiratory drive current; (iv) an increase in frequency and amplitude of sEPSCs; and (v) an increase in respiratory frequency and tonic/seizure-like activity in XIIn rhythmic motor output. These data suggest that a substantial fraction of the nAChRs mediating the nicotinic effects on inspiratory neurons in the preBötC rhythmogenic network contain $\alpha 4$ subunits. Nicotine, via activation of $\alpha 4^{\star}$ nAChRs, facilitates glutamatergic excitatory input to preBötC inspiratory neurons and depolarizes these neurons, resulting in increased respiratory frequency. (3) Microinjection of nicotine at low nanomolar concentrations into the preBötC in L9'A slices increased respiratory frequency, and injection into the ipsilateral XII nucleus induced tonic/seizurelike activity. These results suggest that functional $\alpha 4^{*} \mathrm{nAChRs}$ are present in the preBötC and XII nucleus. Because nicotine at these low concentrations does not activate non- $\alpha 4^{*}$ nAChRs, we conclude that activation of $\alpha 4^{*}$ nAChRs in the preBötC is sufficient for nicotine-induced increase in respiratory rhythm and also that activation of $\alpha 4^{\star}$ receptors in XII nucleus is sufficient for the nicotine-induced increase in tonic motor activity in XIIn. (4) The 
$\alpha 4^{\star}$-selective nAChR antagonist $\mathrm{DH} \beta \mathrm{E}$ blocked the effects of nicotine, suggesting that $\alpha 4^{\star} \mathrm{nAChRs}$ mediate the nicotine effect. Furthermore, in L9'A mice, $\mathrm{DH} \beta \mathrm{E}$ alone induced effects opposite to those of nicotine on preBötC inspiratory neurons and respiratory-related XIIn rhythmic activity. $\mathrm{DH} \beta \mathrm{E}$ induced (i) an outward current associated with a decrease in membrane noise; (ii) a decrease in sEPSC frequency and amplitude; and (iii) a decrease in respiratory frequency. These effects of $\mathrm{DH} \beta \mathrm{E}$ suggest that the hypersensitive receptors are continually activated by endogenous ACh (Shao and Feldman, 2007b). In summary, the results of this study suggest that $\alpha 4^{\star} \mathrm{nAChRs}$ in the preBötC play key functional roles in mediating cholinergic/nicotinic modulation of the excitability of inspiratory neurons and modulation of respiratory rhythm. $\alpha 4^{\star} \mathrm{nAChRs}$ in the XII nucleus play key roles in mediating cholinergic/nicotinic modulation of tonic activity in XIIn. These results are consistent with our previous pharmacological study (Shao and Feldman, 2002) and provide further evidence for the molecular composition of the nAChRs mediating the cholinergic/nicotinic modulation of respiratory pattern. We do not exclude the possibility that other subtypes of nAChRs also exist in the preBötC and XII nucleus and play a role in cholinergic modulation of the respiratory pattern.

We comment on the subunit composition of the $\alpha 4^{\star}$ receptors studied here. Our oocyte expression methods were optimized for expression of $\alpha 4 \mathrm{~L}^{\prime}$ 'A receptors with $(\alpha 4)_{2}(\beta 2)_{3}$ stoichiometry (X. Xiu, N. Puskar, D. Dougherty, and H. A. Lester, unpublished results). Wild-type receptors and $\alpha 4-\mathrm{M} 2$ mutant receptors exist in both $(\alpha 4)_{2}(\beta 2)_{3}$ and $(\alpha 4)_{3}(\beta 2)_{2}$ forms, in mice as well as in heterologous expression systems. These two forms of $\alpha 4 \beta 2$ receptors have distinct sensitivities to both agonists and antagonists. The $(\alpha 4)_{2}(\beta 2)_{3}$ stoichiometry is thought to display the higher sensitivity to $\mathrm{ACh}$, nicotine, and $\mathrm{DH} \beta \mathrm{E}$ (Moroni et al., 2006). The substantial effects of presumably modest extracellular ACh levels, and the nearly complete blockade by $0.2 \mu \mathrm{M} \mathrm{DH} \beta \mathrm{E}$, suggest that the nAChRs underlying the nicotinic modulation of respiratory pattern, detected in our experiments, are the highsensitivity $(\alpha 4)_{2}(\beta 2)_{3}$ type. However, because other subunits may also be present in the preBötC or XII nucleus, it is premature to make definite conclusions about the precise stoichiometry. For example, $\alpha 4 \beta 4^{\star}$ receptors are also very sensitive to blockade by $\mathrm{DH} \beta \mathrm{E}$. However, $\beta 4$ subunit expression has not been seen in these regions in neonatal and adult rats (DineleyMiller and Patrick, 1992; Zoli et al., 1995; Winzer-Serhan and Leslie, 1997).

\section{Nicotine alters the excitability of preBötC inspiratory neurons via $\alpha 4^{\star}$ nAChRs}

Taking advantage of the genetically engineered L9'A mouse strain, we were able to activate specifically the $\alpha 4^{*} \mathrm{nAChRs}$ with low nanomolar concentrations of nicotine (Tapper et al., 2004; Fonck et al., 2005). Because the sEPSCs and the phasic inspiratory drive current are not blocked by $\mathrm{DH} \beta \mathrm{E}$ or other AChR antagonists [such as nicotinic antagonists mecamylamine, methyllycaconitine, and muscarinic antagonist atropine (Shao and Feldman, 2000, 2001, 2002)] but can be completely blocked by 6-cyano-7-nitroquinoxaline-2,3-dione, they are glutamatergic EPSCs from excitatory synapses on the preBötC inspiratory neurons (Shao and Feldman, 2001). The results in this study that nicotine at low nanomolar concentrations increased the frequency and amplitude of sEPSCs, whereas $\mathrm{DH} \beta \mathrm{E}$ decreased them in L9'A mice, suggest that nicotine or ambient levels of ACh [volume transmission (Zoli et al., 1999; Dani and Bertrand, 2007)] facilitate glutamatergic transmission by activation of $\alpha 4^{*}$
nAChRs on presynaptic sites and/or upstream to the synaptic boutons [including preterminal nAChRs (Lena et al., 1993)] of neurons that send excitatory input to preBötC inspiratory neurons. The results that nicotine induced a tonic inward current associated with an increase in membrane noise in inspiratory neurons could arise from activation of postsynaptic and/or extrasynaptic nAChRs in addition to facilitation of glutamatergic transmission. Our results in current study do not exclude the possibility that activation of postsynaptic and/or extrasynaptic $\alpha 4^{\star}$ nAChRs by nicotine or by ambient levels of ACh may also contribute to modulation of the excitability of the postsynaptic neurons. Increase in excitability and depolarization of preBötC inspiratory neurons results in an increase in respiratory frequency.

Nicotine sensitivity of wild-type rodents and L9' mutant mice Our data indicate that nicotine is less potent in modulating respiratory rhythm in neonatal C57BL/6 mice (wild-type) than Sprague Dawley rats of similar ages. In Sprague Dawley neonatal rats, respiratory frequency in slices increases by $188 \pm 28 \%$ with $0.2 \mu \mathrm{M}$ nicotine and by $280 \pm 61 \%$ with $0.5 \mu \mathrm{M}$ nicotine (Shao and Feldman, 2001). In wild-type C57BL/6 neonatal mice in this study, respiratory frequency increased by $119.1 \pm 15.9 \%$ with 0.5 $\mu \mathrm{M}$ and by $147.7 \pm 19.8 \%$ with $1 \mu \mathrm{M}$ nicotine (Fig. $1 C$ ). There are clearly species differences in cholinergic/nicotinic modulation of respiratory rhythm.

Although nicotine is less potent in wild-type mice, the L9' A mutation on the $\alpha 4^{\star}$ nAChRs renders nicotine $\sim 100$-fold more potent to induce cellular and systemic respiratory responses. This increase in sensitivity agrees well with similar increases measured for L9'A receptors expressed in oocytes (Fonck et al., 2005), for nicotine-induced $\mathrm{Ca}^{2+}$ increases in neurons from $\mathrm{L9}^{\prime} \mathrm{A}$ mice (Tapper et al., 2004; Fonck et al., 2005), and for nicotine-induced hypothermia in L9' A mice (Tapper et al., 2004, 2007). In neonatal wild-type rats, application of $\mathrm{DH} \beta \mathrm{E}$ alone does not affect preBötC neurons and the respiratory rhythm, probably because of low concentrations of background ACh release under in vitro conditions (Shao and Feldman, 2005). However, in L9' A mice, $\mathrm{DH} \beta \mathrm{E}$ induced effects opposite to nicotine application, suggesting that the L9' A nAChRs are sensitive to the endogenous ambient levels of ACh (Shao and Feldman, 2007b) and that these $\alpha 4^{*}$ $n A C h R s$ are not completely desensitized by the endogenous ligands, consistent with the finding that the hypersensitive receptors have a "window" of activation in the sustained presence of agonist (Fonck et al., 2005). Therefore, blockade of these L9' A receptors antagonizes the endogenous $\mathrm{ACh}$ actions on preBötC inspiratory neurons and the respiratory rhythm, resulting in opposite effects. This rather subtle effect of $\mathrm{DH} \beta \mathrm{E}$ is the first report that endogenous ACh alone has a detectable effect in L9' A mice at the cellular level. However, L9' A mice do have a sleep disorder that could arise from endogenous ACh (Fonck et al., 2005). An even more hypersensitive strain, L9'S, is so hypersensitive to endogenous agonists that their seizure threshold to anticholinesterase drugs is significantly lower than that of wild-type mice (Fonck et al., 2003).

\section{nAChRs in XII nucleus}

Hypoglossal motoneurons innervate the tongue and upper airway muscles (Aldes, 1995; Dobbins and Feldman, 1995) and play an important role in regulating upper airway resistance and patency (Fuller et al., 1999). Immunoreactivity for $\alpha 4$ and $\alpha 7$ $\mathrm{nAChR}$ subunits is present in the XII nucleus (Dominguez del Toro et al., 1994; Dehkordi et al., 2005). $\beta 2$ subunit mRNA is also 
detectable (Wada et al., 1989). Activation of nAChRs excites hypoglossal motoneurons (Zaninetti et al., 1999; Chamberlin et al., 2002; Robinson et al., 2002; Quitadamo et al., 2005). In this study, we illustrated that functional $\alpha 4^{\star} \mathrm{nAChRs}$ were expressed in the XII nucleus and that selective activation of these receptors enhanced tonic activity without affecting the amplitude and duration of inspiratory bursts of XIIn motor output. Activation of $\alpha 4^{*}$ nAChRs likely depolarizes hypoglossal motoneurons (Zaninetti et al., 1999; Chamberlin et al., 2002), resulting in tonic spiking that underlies the tonic/seizure-like activity in XIIn. These results suggest that nAChRs in the XII nucleus play key functional roles in modulating the respiratory-related motor activity of XIIn, whereas $\alpha 4^{\star}$ nAChRs primarily contribute to the mediation of the cholinergic regulation of upper airway tone. These results (Fig. 6) are consistent with those of Fonck et al. (2005) and suggest that cholinergic modulation of motoneuronal excitability via $\alpha 4^{*}$ nAChRs may, partially, underlie hypersensitivity of L9'A mice to nicotine-induced seizures.

\section{References}

Aldes LD (1995) Subcompartmental organization of the ventral (protrusor) compartment in the hypoglossal nucleus of the rat. J Comp Neurol 353:89-108.

Anderson ME, Johnson DC, Batal HA (2005) Sudden Infant Death Syndrome and prenatal maternal smoking: rising attributed risk in the Back to Sleep era. BMC Med 3:4.

Bellingham MC, Ireland MF (2002) Contribution of cholinergic systems to state-dependent modulation of respiratory control. Respir Physiol Neurobiol 131:135-144.

Chamberlin NL, Bocchiaro CM, Greene RW, Feldman JL (2002) Nicotinic excitation of rat hypoglossal motoneurons. Neuroscience 115:861-870.

Champtiaux N, Changeux JP (2004) Knockout and knockin mice to investigate the role of nicotinic receptors in the central nervous system. Prog Brain Res 145:235-251.

Clements JD, Bekkers JM (1997) Detection of spontaneous synaptic events with an optimally scaled template. Biophys J 73:220-229.

Dani JA, Bertrand D (2007) Nicotinic acetylcholine receptors and nicotinic cholinergic mechanisms of the central nervous system. Annu Rev Pharmacol Toxicol 47:699-729.

Dehkordi O, Haxhiu MA, Millis RM, Dennis GC, Kc P, Jafri A, Khajavi M, Trouth CO, Zaidi SI (2004) Expression of alpha-7 nAChRs on spinal cord-brainstem neurons controlling inspiratory drive to the diaphragm. Respir Physiol Neurobiol 141:21-34.

Dehkordi O, Millis RM, Dennis GC, Coleman BR, Johnson SM, Changizi L, Ovid Trouth C (2005) Alpha-7 and alpha-4 nicotinic receptor subunit immunoreactivity in genioglossus muscle motoneurons. Respir Physiol Neurobiol 145:153-161.

Dineley-Miller K, Patrick J (1992) Gene transcripts for the nicotinic acetylcholine receptor subunit, beta4, are distributed in multiple areas of the rat central nervous system. Brain Res Mol Brain Res 16:339-344.

Dobbins EG, Feldman JL (1995) Differential innervation of protruder and retractor muscles of the tongue in rat. J Comp Neurol 357:376-394.

Dominguez del Toro E, Juiz JM, Peng X, Lindstrom J, Criado M (1994) Immunocytochemical localization of the $\alpha 7$ subunit of the nicotinic acetylcholine receptor in the rat central nervous system. J Comp Neurol 349:325-342.

Feldman JL, Mitchell GS, Nattie EE (2003) Breathing: rhythmicity, plasticity, chemosensitivity. Annu Rev Neurosci 26:239-266.

Fonck C, Nashmi R, Deshpande P, Damaj MI, Marks MJ, Riedel A, Schwarz J, Collins AC, Labarca C, Lester HA (2003) Increased sensitivity to agonist-induced seizures, Straub tail, and hippocampal theta rhythm in knock-in mice carrying hypersensitive $\alpha 4$ nicotinic receptors. J Neurosci 23:2582-2590.

Fonck C, Cohen BN, Nashmi R, Whiteaker P, Wagenaar DA, RodriguesPinguet N, Deshpande P, McKinney S, Kwoh S, Munoz J, Labarca C, Collins AC, Marks MJ, Lester HA (2005) Novel seizure phenotype and sleep disruptions in knock-in mice with hypersensitive $\alpha 4^{\star}$ nicotinic receptors. J Neurosci 25:11396-11411.

Fuller DD, Williams JS, Janssen PL, Fregosi RF (1999) Effect of co- activation of tongue protrudor and retractor muscles on tongue movements and pharyngeal airflow mechanics in the rat. J Physiol (Lond) 519:601-613

Gotti C, Riganti L, Vailati S, Clementi F (2006) Brain neuronal nicotinic receptors as new targets for drug discovery. Curr Pharm Des 12:407-428.

Gray PA, Rekling JC, Bocchiaro CM, Feldman JL (1999) Modulation of respiratory frequency by peptidergic input to rhythmogenic neurons in the preBötzinger complex. Science 286:1566-1568.

Harvey SC, Luetje CW (1996) Determinants of competitive antagonist sensitivity on neuronal nicotinic receptor $\beta$ subunits. J Neurosci 16:3798-3806.

Harvey SC, Maddox FN, Luetje CW (1996) Multiple determinants of dihydro- $\beta$-erythroidine sensitivity on rat neuronal nicotinic receptor $\alpha$ subunits. J Neurochem 67:1953-1959.

Kinney HC, Filliano JJ, Sleeper LA, Mandell F, Valdes-Dapena M, White WF (1995) Decreased muscarinic receptor binding in the arcuate nucleus in sudden infant death syndrome. Science 269:1446-1450.

Labarca C, Schwarz J, Deshpande P, Schwarz S, Nowak MW, Fonck C, Nashmi R, Kofuji P, Dang H, Shi W, Fidan M, Khakh BS, Chen Z, Bowers BJ, Boulter J, Wehner JM, Lester HA (2001) Point mutant mice with hypersensitive $\alpha 4$ nicotinic receptors show dopaminergic deficits and increased anxiety. Proc Natl Acad Sci USA 98:2786-2791.

Lena C, Changeux JP, Mulle C (1993) Evidence for "preterminal" nicotinic receptors on GABAergic axons in the rat interpeduncular nucleus. J Neurosci 13:2680-2688.

Liu G, Feldman JL, Smith JC (1990) Excitatory amino acid-mediated transmission of inspiratory drive to phrenic motoneurons. J Neurophysiol 64:423-436.

Mitchell EA, Milerad J (2006) Smoking and the sudden infant death syndrome. Rev Environ Health 21:81-103.

Moroni M, Zwart R, Sher E, Cassels BK, Bermudez I (2006) $\alpha 4 \beta 2$ nicotinic receptors with high and low acetylcholine sensitivity: pharmacology, stoichiometry, and sensitivity to long-term exposure to nicotine. Mol Pharmacol 70:755-768.

Nicholson C (1985) Diffusion from an injected volume of a substance in brain tissue with arbitrary volume fraction and tortuosity. Brain Res 333:325-329.

Picciotto MR, Caldarone BJ, Brunzell DH, Zachariou V, Stevens TR, King SL (2001) Neuronal nicotinic acetylcholine receptor subunit knockout mice: physiological and behavioral phenotypes and possible clinical implications. Pharmacol Ther 92:89-108.

Quitadamo C, Fabbretti E, Lamanauskas N, Nistri A (2005) Activation and desensitization of neuronal nicotinic receptors modulate glutamatergic transmission on neonatal rat hypoglossal motoneurons. Eur J Neurosci 22:2723-2734.

Robinson DM, Peebles KC, Kwok H, Adams BM, Clarke LL, Woollard GA, Funk GD (2002) Prenatal nicotine exposure increases apnoea and reduces nicotinic potentiation of hypoglossal inspiratory output in mice. J Physiol (Lond) 538:957-973.

Shao XM, Feldman JL (2000) Acetylcholine modulates respiratory pattern: effects mediated by M3-like receptors in preBötzinger complex inspiratory neurons. J Neurophysiol 83:1243-1252.

Shao XM, Feldman JL (2001) Mechanisms underlying regulation of respiratory pattern by nicotine in preBötzinger Complex. J Neurophysiol $85: 2461-2467$.

Shao XM, Feldman JL (2002) Pharmacology of nicotinic receptors in preBötzinger Complex that mediate modulation of respiratory pattern. J Neurophysiol 88:1851-1858.

Shao XM, Feldman JL (2005) Cholinergic neurotransmission in the preBötzinger Complex modulates excitability of inspiratory neurons and regulates respiratory rhythm. Neuroscience 130:1069-1081.

Shao XM, Feldman JL (2007a) Micro-agar salt bridge in patch-clamp electrode holder stabilizes electrode potentials. J Neurosci Methods 159:108-115.

Shao XM, Feldman JL (2007b) Efficient measurement of endogenous neurotransmitters in small localized regions of central nervous systems in vitro with HPLC. J Neurosci Methods 160:256-263.

Smith JC, Ellenberger HH, Ballanyi K, Richter DW, Feldman JL (1991) PreBötzinger complex: a brainstem region that may generate respiratory rhythm in mammals. Science 254:726-729.

Tapper AR, McKinney SL, Nashmi R, Schwarz J, Deshpande P, Labarca C, 
Whiteaker P, Marks MJ, Collins AC, Lester HA (2004) Nicotine activation of $\alpha 4^{*}$ receptors: sufficient for reward, tolerance, and sensitization. Science 306:1029-1032.

Tapper AR, McKinney SL, Marks MJ, Lester HA (2007) Nicotine responses in hypersensitive and knockout $\alpha 4$ mice account for tolerance to both hypothermia and locomotor suppression in wild-type mice. Physiol Genomics 31:422-428.

Wada E, Wada K, Boulter J, Deneris E, Heinemann S, Patrick J, Swanson LW (1989) Distribution of alpha 2, alpha 3, alpha 4, and beta 2 neuronal nicotinic receptor subunit mRNAs in the central nervous system: a hybridization histochemical study in the rat. J Comp Neurol 284:314-335.

Whiteaker P, Cooper JF, Salminen O, Marks MJ, McClure-Begley TD, Brown RW, Collins AC, Lindstrom JM (2006) Immunolabeling demonstrates the interdependence of mouse brain $\alpha 4$ and $\beta 2$ nicotinic acetylcholine receptor subunit expression. J Comp Neurol 499:1016-1038.

Winzer-Serhan UH, Leslie FM (1997) Codistribution of nicotinic acetylcholine receptor subunit $\alpha 3$ and $\beta 4$ mRNAs during rat brain development. J Comp Neurol 386:540-554.

Zaninetti M, Tribollet E, Bertrand D, Raggenbass M (1999) Presence of functional neuronal nicotinic acetylcholine receptors in brainstem motoneurons of the rat. Eur J Neurosci 11:2737-2748.

Zoli M, Le Novere N, Hill Jr JA, Changeux JP (1995) Developmental regulation of nicotinic ACh receptor subunit mRNAs in the rat central and peripheral nervous systems. J Neurosci 15:1912-1939.

Zoli M, Jansson A, Sykova E, Agnati LF, Fuxe K (1999) Volume transmission in the CNS and its relevance for neuropsychopharmacology. Trends Pharmacol Sci 20:142-150. 